\section{Detection and Molecular Mass Determination of an HIV Replication- Enhancing Female Genital Tract Factor Using a Blot Bioassay}

BioTechniques 28:478-486 (March 2000)

\begin{abstract}
We previously reported that cervicovaginal lavages $(C V L)$ contain a factor that enhances the replication of human immunodefeciency virus (HIV) by increasing virus transcription in $T$ cells and monocytoid cells. This factor was named the HIV-inducing factor (HIF). To determine the molecular mass of HIF, we adapted a blot technique that involves nonreducing SDS-PAGE of CVL samples and electrophoretic transfer onto nitrocellulose paper followed by incubation of paper slices with HIV-infected monocytoid U1 cells. The slices with HIF bioactivity were detected by increased HIV production and measured by an HIV core protein (p24) ELISA. We refer to this technique as the "BioBlot" assay. The BioBlot assay successfully detected bioactivity of HIF anchored onto nitrocellulose and determined that HIF has a molecular mass of about 14 kDa. Paper slices with HIF-negative CVL samples as well as nitrocellulose paper samples without CVL did not enhance HIV production. This finding suggested that SDS-PAGE and nitrocellulose binding do not functionally alter the bioaccontained an $\mathrm{HIV}$-inducing factor (HIF) which enhanced HIV replication up to 70 -fold (10). The increased expression of HIV was not due to increased cell proliferation as shown by thymidine uptake studies (10). HIF induces HIV expression in both monocytoid and $T$ cells through NFK-B and TRE-mediated activation of the HIVLTR (2). Although HIF has been partially characterized (10), the molecular mass and the structure of HIF is not known. In this report, we describe a novel method that can be used for direct detection of HIF in secretions of the genital tract, as well as determining the molecular mass of HIF. We refer to this method as the "BioBlot" technique for HIV induction, which is a modified version of the "Cell Blot" described by Carnow et al. (3). It basically involves SDS-PAGE of CVL specimens that contain HIF, followed by electrophoretic transfer of the resolved proteins to nitrocellulose paper. The blot is then sliced and transferred to culture plates and seeded with HIV-infected cells to measure HIV-enhancing activity.
\end{abstract} tive domain(s) of HIF structure. In addition to the detection of HIF bioactivity anchored to nitrocellulose and HIF molecular mass determination, the BioBlot technique offers an alternative, rapid method for other applications. These include the study of receptor-ligand interactions of mucosal proteins, direct bioactivity testing and molecular mass determination of secretory substances.

\section{INTRODUCTION}

Human immunodeficiency virus (HIV) is the causative agent of acquired immunodeficiency syndrome (AIDS) afflicting millions of people worldwide (11). Globally, sexual transmission is the major route for HIV infection (1). Mucosal secretions of the genital tract represent an important vehicle for sexual transmission of HIV (5). Factors in mucosal secretions may affect HIV expression by infected cells in the genital tract and thus influence sexual transmission of HIV by increasing the amount of virus in the genital tract (7).

Our laboratory previously reported that $15 \%-20 \%$ of cervicovaginal lavage (CVL) samples, from both HIV seronegative and seropositive women,

\section{MATERIALS AND METHODS}

\section{Cells and Culture Conditions}

The U1 cells were obtained from the AIDS Research and Reference ReagentProgram (contributed by Dr. Thomas Folks), Division of AIDS-NIAID/National Institutes of Health (Rockville, MD, USA). U1 cells are transformed monocytoid cells derived from the U937 cell line and contain a stable copy of the complete HIV- $1_{\text {IIIB }}$ genome (6). U1 cell cultures were fed twice weekly with a complete medium consisting of RPMI-1640 supplemented with $0.01 \mathrm{M}$ HEPES, 0.002 M glutamine and $10 \%$ fetal bovine serum (FBS) (BioWhittakker, Walkerville, MD, USA). Before each experiment, cell viability was checked by trypan blue dye exclusion. The U1 cells were negative for mycoplasma by PCR (9).

\section{Collection and Preparation of CVL}

CVL samples were collected from volunteers in the Women Interagency HIV study (WIHS) at Rush Medical College. For collection of CVL, the cervix and vagina were sequentially irrigated with two $10 \mathrm{~mL}$ aliquots of sterile normal saline, which were then pooled. Specimens of CVL were centrifuged $(700 \times g)$, filtered $(0.45 \mu \mathrm{m}$ pore size) and stored at $-70^{\circ} \mathrm{C}$.

\section{Detection of Bioactivity in CVL Samples}

HIF activity was measured as previously described (10). Approximately $2 \times 10^{4}$ U1 cells were transferred to microplates in $190 \mu \mathrm{L}$ of complete medium. CVL specimens were heated at $100^{\circ} \mathrm{C}$ for $5 \mathrm{~min}$, and $10 \mu \mathrm{L}$ were added to the culture to give a total volume of $200 \mu \mathrm{L}$. After $72 \mathrm{~h}$, levels of p24 in the culture were determined by ELISA (SAIC Frederick, NCI-Frederick Cancer Research and Development Center, Frederick, MD, USA) (10). Before measuring the p24 concentration, culture supernates were treated with $0.5 \%$ Triton ${ }^{\circledR}$ X-100 (Sigma, St. Louis, MO, USA). Positive controls included TNF$\alpha(50 \mathrm{U} /$ well) and phorbal myristate acetate (PMA) at $25 \mathrm{ng} / \mathrm{mL}$. Negative controls consisted of complete media alone, sterile nitrocellulose paper and nitrocellulose paper from a region outside the protein blot area. The same (+) and (-) CVL samples were used throughout these studies. The protein concentration of (+) and (-) CVL samples were $18 \mu \mathrm{g} / \mathrm{mL}$ and $92 \mu \mathrm{g} / \mathrm{mL}$, respectively, determined by BCA protein assay (Pierce Chemical, Rockford, IL, USA). Testing of 32 CVL samples did not reveal any correlation between total protein in CVL samples and their HIF bioactivity (data not shown). 


\section{Short Technical Reports}

Detection of HIF Activity in CVL Sample Directly Applied to Nitrocellulose Paper

The center of two $5 \times 5 \mathrm{~cm}$ pieces of nitrocellulose paper were marked by a sterile pipet tip, and each piece received a total $100 \mu \mathrm{L}$ of HIF positive or HIF negative CVL samples. First, $50 \mu \mathrm{L}$ of CVL were added and allowed to dry under sterile conditions in a laminar flow hood. Then, an additional $50 \mu \mathrm{L}$ were added to the center of the paper and allowed to dry. The central region of the papers were then cut to three $1 \times$ $1 \mathrm{~cm}$ pieces and were individually placed in the wells of a 48-well culture plate (Corning, New York, NY, USA). The U1 bioassay was carried out as described below.

\section{Electrophoresis}

CVL samples were separated in Tris-glycine SDS-PAGE as described by Weber and Osborn (12). Samples were treated with electrophoresis sample buffer $(0.0625 \mathrm{M}$ Tris- $\mathrm{HCl}, 2.3 \%$ SDS, glycerol and $0.05 \%$ bromophenol blue ( $\mathrm{pH} \mathrm{6.8)}$ and heated at $70^{\circ} \mathrm{C}$ for 10 min in the absence of $5 \% \beta$-mercap- toethanol. Samples were then loaded on a $12 \%$ SDS-polyacrylamide slab gel (gel buffer $=0.375 \mathrm{M}$ Tris-HCl, $0.1 \%$ SDS, $\mathrm{pH} 8.8$ ) with a $3 \%$ stacking gel (gel buffer $=0.125 \mathrm{M}$ Tris-HCl, $0.1 \%$ SDS, pH 6.8). For each well, $300 \mu \mathrm{L}$ of CVL samples corresponded to $5.4 \mu \mathrm{g}$ protein of the HIF (+) sample and 27.5 $\mu \mathrm{g}$ protein of the HIF (-) sample. Electrophoresis was carried out for $4 \mathrm{~h}$ at $120 \mathrm{~V}$ using a dual vertical slab apparatus (Bio-Rad Laboratories, Hercules, CA, USA) with cooling in an electrophoresis buffer that contained of $0.05 \mathrm{M}$ Tris, $0.192 \mathrm{M}$ glycine and $0.1 \%$ SDS, pH 8.3. The apparent molecular masses of the separating proteins were determined using prestained molecular weight markers (Rainbow, Amersham Corporation, UK) that consisted of 200 $\mathrm{kDa}$ myosin, $92.5 \mathrm{kDa}$ phosphorylase b, $69 \mathrm{kDa}$ BSA, $46 \mathrm{kDa}$ ovalbumin, 30 $\mathrm{kDa}$ carbonic anhydrase, $21.5 \mathrm{kDa}$ trypsin inhibitor and $14.3 \mathrm{kDa}$ lysozyme.

\section{Electroblotting and Silver Staining}

After SDS-PAGE, the proteins were electrophoretically transferred onto nitrocellulose paper (Bio-Rad Laborato- ries) as described previously (8). The blotting buffer consisted of $0.5 \mathrm{M}$ Tris, $0.38 \mathrm{M}$ glycine, $0.01 \%$ SDS and $10 \%$ methanol, pH 8.3. The transfer was performed in a blotting apparatus (Hoeffer Scientific Instruments, San Fransisco, CA, USA) at $1 \mathrm{~mA}$ for $90 \mathrm{~min}$ at $4^{\circ} \mathrm{C}$. After the transfer, the blot papers were washed $3 \times$ with $20 \mathrm{~mL}$ of sterile PBS. The blot papers were then sliced into fifteen $7.5 \mathrm{~mm}$ sections and transferred to a 48 -well culture plate to measure HIV induction activity in U1 cells. Duplicate SDS-PAGE gels that were run simultaneously were stained using a silver staining kit (Bio-Rad Laboratories).

\section{Biological Assays for Electroblotted CVL Sample}

To test HIF activity of electroblotted CVL sample on nitrocellulose paper, $8 \times 10^{4} \mathrm{U} 1$ cells in $0.5 \mathrm{~mL}$ complete medium were incubated with slices of nitrocellulose paper in wells of 48 -well plates. After $72 \mathrm{~h}$ of incubation at $37^{\circ} \mathrm{C}$, culture supernatant fluids were collected and, as an indicator of HIV expression, HIV core protein p24 was measured as described above.

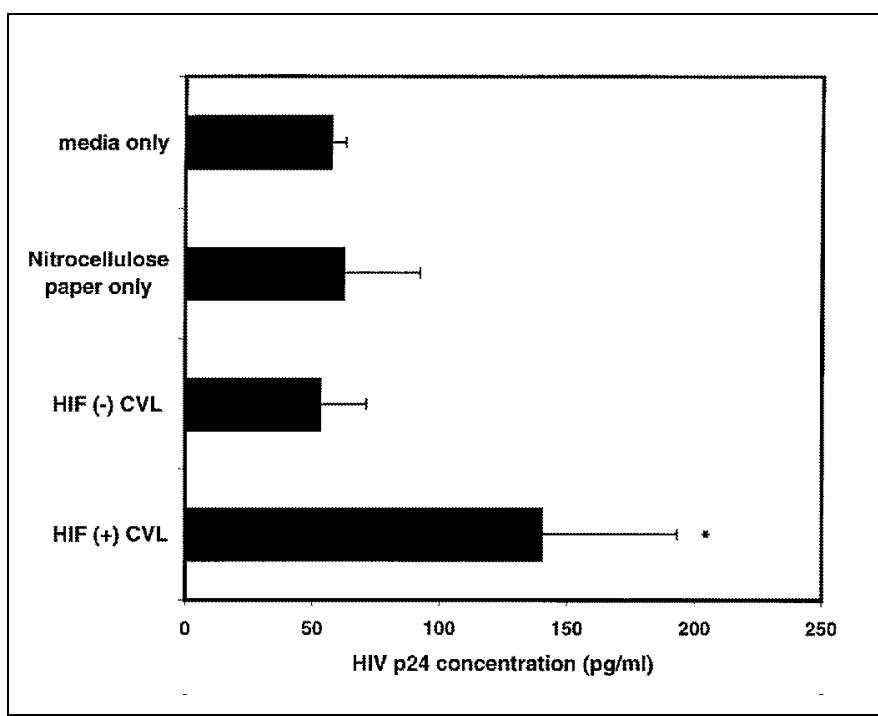

Figure 1. Bioactivity of CVL sample directly applied to nitrocelllose paper. Pieces of nitrocellulose paper, loaded with $150 \mu \mathrm{L}$ of CVL sample, were incubated with U1 cells. After $72 \mathrm{~h}$, HIV production was measured by release of p24 into culture medium. The HIF positive CVL sample shows threefold higher HIV p24 levels than either the negative CVL or nitrocellulose paper only $(P=0.049$, unpaired t-test $)$. Data represent two experiments using triplicate wells. Sample designated as $(+)$ CVL is defined as a sample that induces 24 production by $\mathrm{U} 1$ cells $\geq$ fivefold over the basal levels. Samples that do not induce p 24 production above fivefold are referred to as (-) CVL.

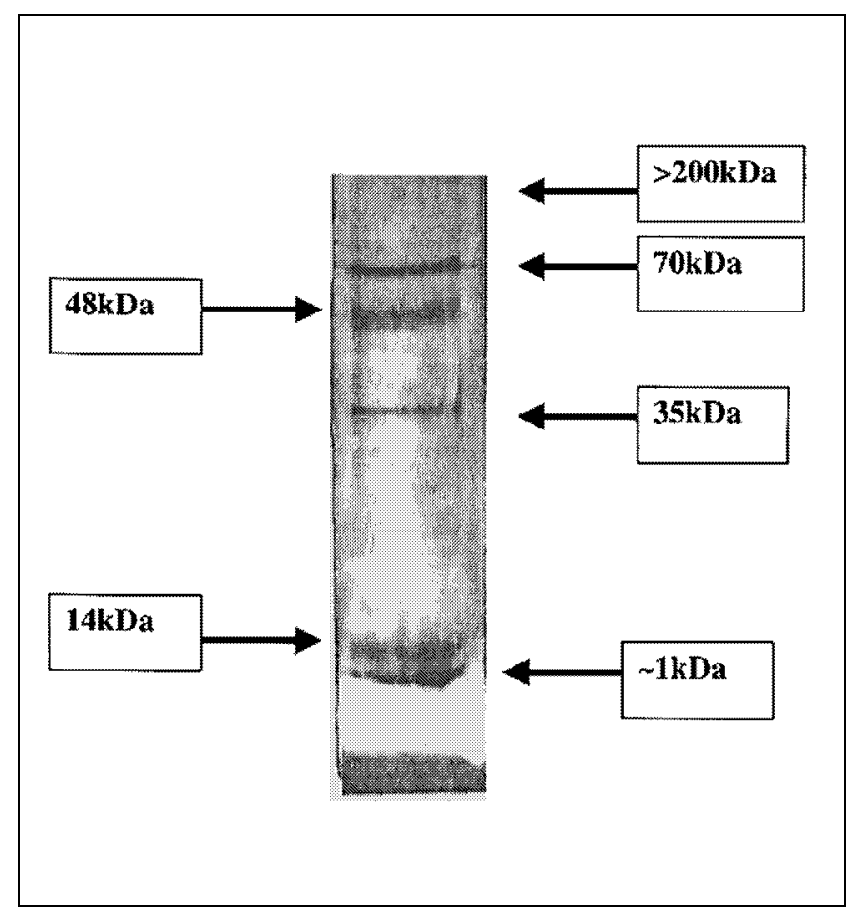

Figure 2. Nonreducing SDS-PAGE analysis of semipurified HIF positive CVL sample. CVL specimen $(300 \mu \mathrm{L})$ was loaded on a nonreducing SDS-PAGE gel (12\%). The gel was silver stained and showed five distinct bands. 


\section{Short Technical Reports}

\section{RESULTS}

\section{Detection of HIF after Direct Appli- cation of CVL Samples to Nitrocellulose Paper}

To determine whether the bioactivity of HIF bound to nitrocellulose can be detected, the CVL samples with or without HIF bioactivity were directly applied onto nitrocellulose paper. The resulting loaded blots were then added to cultures of chronically HIV-infected $\mathrm{U} 1$ cells. These cells have been used as a model to test the effects of various substances including cytokines (4) on HIV replication in monocytoid cells. Before the BioBlot assay, the HIF positive (final concentrations of $0.5 \%$ ) and negative CVL samples were tested for bioactivity.

Figure 1 shows that the addition of blot paper with the HIF positive CVL sample increased HIV production by about threefold, while untreated U1 cells produced low levels of HIV. The culture wells that received HIF negative CVL sample or untreated nitrocellulose paper induced levels of p24 protein similar to those of untreated cells. (Figure 1).

\section{Determination of the Molecular Mass of HIF by BioBlot Assay}

To identify the molecular mass of proteins that correspond to HIF activity, HIF positive and negative CVL samples were analyzed by SDS-PAGE, followed by silver staining on a separate gel (sensitivity: $5 \mathrm{ng}$ protein per well). Five distinct visible protein bands were observed in both CVL samples, with apparent molecular masses of approximately $70,48,35,14$ and $1 \mathrm{kDa}$ at the migration front (Figure 2). Unresolved high-molecular-weight aggregates were also present on top of the gel (not shown). None of the visible bands was unique to the $\mathrm{HIF}(+) \mathrm{CVL}$ sample. This band pattern was similar to patterns previously observed using 30 different (+) CVL samples (not shown).

To determine the bioactive bands, two CVL samples, with and without HIF bioactivity, were run on a nonreducing SDS-PAGE gel and followed by electroblotting of the samples as described above. After incubation of U1 cells with blot paper, slices from two adjacent regions of the HIF positive CVL blot, corresponding to molecular mass of approximately 16 and $14 \mathrm{kDa}$, showed the highest HIV enhancing activity (Figure 3 ). The degree of HIV enhancement was 8-fold and 15-fold higher than that of the untreated U1 cells. Blot slices from other sections of the HIF positive CVL sample showed near basal levels of HIF bioactivity. Moreover, blot slices from all regions of the HIF negative CVL sample, as well as regions of the blot that were not treated with CVL sample, showed no induction of HIV in U1 cells. The results in Figure 3 show that the BioBlot technique was able to detect HIF bioactivity after the SDS-PAGE/electroblotting procedures and that HIF has a molecular mass of $14 \mathrm{kDa}$.

\section{DISCUSSION}

We have developed a novel method for two potentially important applications: first, the detection of an HIF found in the female genital tract secre- tions and second, the molecular mass determination of HIF. This is the first report to show the usefulness of the BioBlot assay technique for measuring bioactivity of HIV activating factors and molecular weight determinations of proteins from genital tract secretions.

The BioBlot assay is similar to the Cell Blot technique developed by Carnow et al. (7) and makes use of the electrophoretic mobility of ciliary neurotrophic factors by staining live neurons growing in a region on nitrocellulose paper. The Cell Blot technique has also been used to study molecules such as nerve growth factors (8). The ability to detect HIF bioactivity after binding to nitrocellulose paper suggests that functional sites of HIF are available and that protein refolding during denaturation and SDS treatment does not abolish the HIF bioactivity. Because protein binding to nitrocellulose paper is stable and difficult to reverse without harsh enzymatic treatment, the detection of HIF bioactivity anchored to nitrocellulose paper also suggests that HIF can exert its HIV-inducing effects

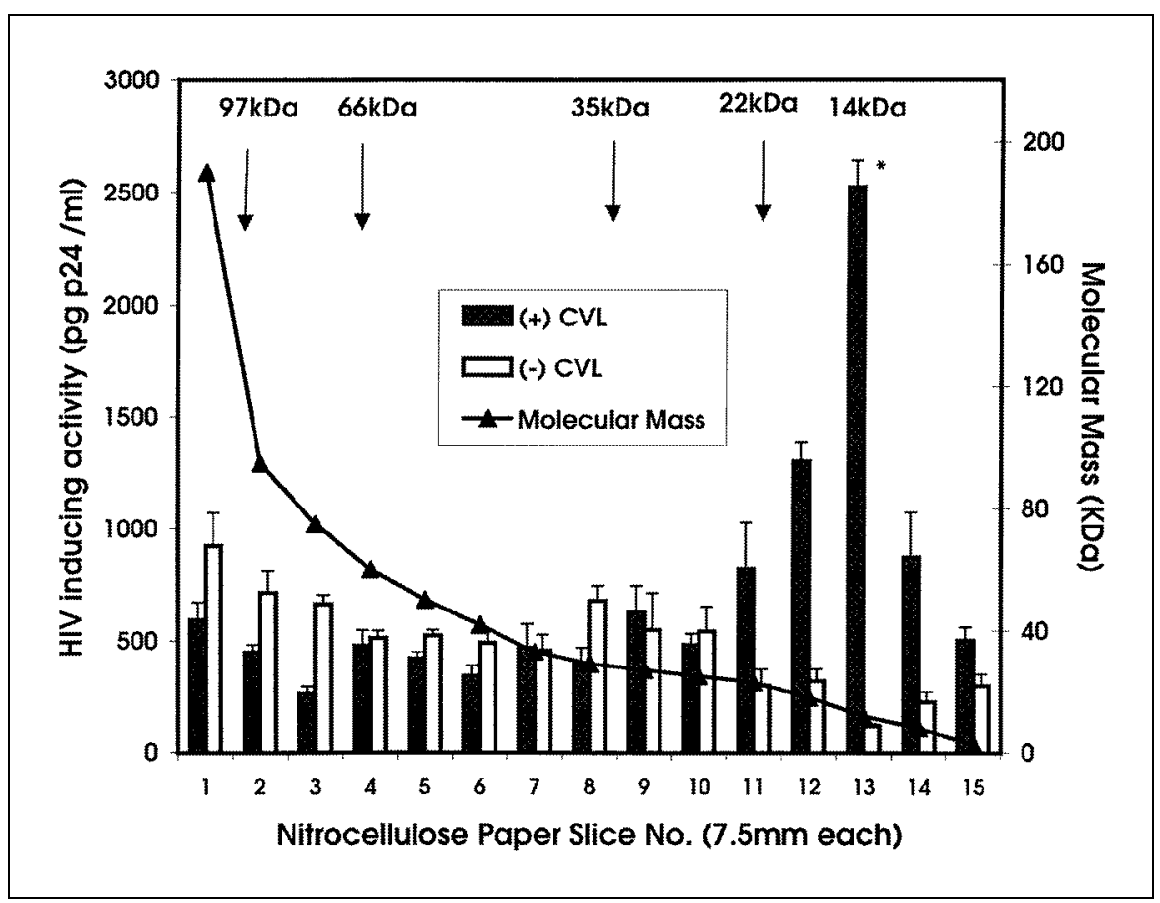

Figure 3. Molecular mass determination of HIF by analysis of CVL samples electroblotted onto nitrocellulose paper. CVL samples with and without the activity were transferred onto nitrocellulose by electroblotting after nonreducing SDS-PAGE. The paper was cut into $7.5 \mathrm{~mm}$ sections and incubated with $\mathrm{U} 1$ cells. After $72 \mathrm{~h}$, culture supernatant fluids were evaluated for $\mathrm{p} 24$ production. Peaks of p24 production identify the apparent molecular mass of HIF. For definitions of (+) CVL and (-) CVL, refer to Figure 1 legend. Data is representative of two experiments carried out in triplicate culture wells. $[P=0.0003, P=0.0004$ unpaired t-test, compared to (-) $\mathrm{CVL}$ and medium alone, respectively.] 


\section{Short Technical Reports}

on U1 cells without being internalized by the cells. Furthermore, this observation indicates that HIF may act through a cell-surface receptor.

The BioBlot technique offers many advantages over methods that test a sample's bioactivity directly in solution. For example, in addition to enabling investigators to determine the molecular mass of the compound of interest, it allows for removing possible cytotoxic substances such as inorganic molecules, toxins, proteases and salts or other interfering substances from specimens. This can be simply achieved by binding the protein of interest to nitrocellulose paper with subsequent washing of the blot before bioassays.

\section{REFERENCES}

1.Alexander, N.J. 1990. Sexual transmission of HIV: virus entry into male and female genital tract. Fertil. Steril. 54:1-18.
2.Al-Harthi, L., G.T. Spear, F.B. Hashemi, A. Landay, B. Sha and K.A. Roebuck. 1998.An HIV inducing factor from the genital tract activates HIV-1 gene expression through the k-B enhancer. J. Infec. Dis. 178:1343-1351.

3.Carnow, T.B., M. Manthrope, G.E. Davis and S. Varon. 1985. Localized survival of ciliary ganglionic neurons identifies neurotropic factor bands on nitrocellulose blots. J. Neurosci. 5:1965-1971.

4.Ciacci-Woolwine, F., I.C. Blomfield, S.H. Richardson and S.B. Mizel. 1998. Salmonella flagellin induces tumor necrosis factor alpha in a human promonocytic cell line. Infect. Immunol. 66:1127-1134.

5.De Vincenczi, I. 1994. A longitudinal study of HIV transmission by heterosexual partners. N. Engl. J. Med. 331:341.

6.Folks, T.M., J.S. A. Justement, C.A. Kinter, C.A. Dinarello and A.S. Fauci. 1988. Cytokine-induced expression of HIV-1 in a chronically infected promonocyte cell line. Science 238:800.

7.Milman, G. and O. Sharma. 1994. Mechanisms of HIV/SIV mucosal transmission. AIDS Res. Hum. Retroviruses 10:1305-1312.

8.Pettman, B., M. Manthorpe, J.A. Powell and S. Varon. 1988. Biological activities of nerve growth factor bound to nitrocellulose paper by western blotting. J. Neurosci. 8:3624-3632.

9.Pruckler, J.M. and E.W. Ades. 1995. Detection by polymerase chain reaction of all common Mycoplasma in a cell culture facility. Pathobiology 63:9-11.

10.Spear, G.T., L. Al-Harthi, B. Sha, M.N. Sarloos, M. Hayden, S.L. Massad, C. Benson, K.A. Roebuck and A. Landay. 1997. A potent activator of HIV-1 replication is present in the genital tract of a subset of HIV-1-infected and uninfected women. AIDS 11:1319.

11.The Joint United Nations Program on HIV/AIDS. December 1997. The status and trends of the HIV/AIDS epidemics. Conference at Abidjan, Ivory Coast.

12.Weber, K. and M. Osborn. 1969. The reliability of molecular weight determinations by dodecyl sulfate-polyacrylamide gel electrophoresis. J. Biol. Chem. 244:4406-4412.

We would like to thank Dr. Beverly Sha (Department of Internal Medicine, Section for Infectious Diseases at Rush Medical College) for helping with the collection of CVL samples. Informed consent was granted by the female subjects participating in 
this study, as a part of the Women's Interagency HIV Study (WIHS) at Rush-Presbyterian St. Luke's Medical Center. Human investigation guidelines set forth by the US Department of Health and Human Services and Rush-Presbyterian St. Luke's Medical Center were observed. [Internal Review Board (IRB) approval No. 99031602]. Financial support provided by the WIHS Grant No. 5U01 AI34993-3 and National Institutes of Health Grant No. AI-31812 and Grant No. 2-P50-AR39239 from NIHNIAMS. Address correspondence to Dr. Farhad B. Hashemi, Department of Immunology/Microbiology, Rush University, 1653 W. Congress Pkwy. Chicago, IL 60612 USA.Internet:fhashem2@rush.edu

Received 16 June 1999; accepted 6 October 1999.

Farhad B. Hashemi, Gregory T. Spear, Lawrence Madsen and Juergen Mollenhauer Rush University Chicago, IL, USA

\section{Preparation of Sensitive and Specific Oligonucleotide Probes Tailed Using Terminal Transferase and dITP}

BioTechniques 28:486-490 (March 2000)

\section{ABSTRACT}

An oligonucleotide probe tailed with deoxyadenosine-5'-triphosphate or deoxythymine-5'-triphosphate is detectable with high sensitivity, but has a major drawback-the tail co-hybridizes specifically to complementary sequences. This can be a probem when screening $c D N A$ clones that contain poly(dA) sequences. While it is possible to mask the cDNA tail with unlabeled poly $(d A)$ or poly $(A)$ oligonucleotides, falsepositive clones are still produced because complete masking of extremely long $(d A)$ tails is difficult. As a result, only cDNA clones that have extremely long poly(dA) sequences are often obtained by hybridization screening using tailed probes. In this report, we describe an oligonucleotide probe tailed with DIG-labeled nucleotide in combination with deoxyinosine-5' -triphosphate that was highly specific and sensitive to cDNAs. Terminal deoxynucleotidyl transferase efficiently adds dI nucleotides to the 3 '-end. The dI of the tails did not pair with any nucleotides under stringent hybridization so that the specificity of hybridization assays remained high without affecting the sensitivity of the test. Colony hybridization experiments demonstrated that there were very few ( 1 of 80 tested) false positives using this technique. Its use may increase the accuracy of cDNA screening.

\section{INTRODUCTION}

Labeling of oligonucleotides is important for screening gene libraries using hybridization methods (10). A variety of nonisotopic labeling reactions are used for introducing a labeling molecule to a $3^{\prime}$ - or $5^{\prime}$-end $(3,4,7)$, some internal nucleotides (12) or the $3^{\prime}$-tail (5). Specificity to the oligonucleotide sequence is high when using end labeling; however, the sensitivity is low with nonisotopic labeling. The highest sensitivity is obtained by tailing, using DIG-labeled nucleotide in combination with deoxyadenosine- 5 '-triphosphate (dATP) as substrates of terminal deoxynucleotidyl transferase (TdT); however, the specificity is often reduced due to the added tail. This is especially true in screening cDNA clones: the homopolymeric dA tail hybridizes to $\mathrm{dA} / \mathrm{dT}$ regions in the cDNA clones derived from poly(A) sequences of eukaryotic mRNA (2) even if the regions in the cDNA are masked by the addition of unlabeled poly(dA) oligonucleotides. Thus, there has been no nonisotopic method of labeling an oligonucleotide probe for sensitive and specific cDNA screening using hybridization. In this study, we used deoxyinosine- $5^{\prime}$-triphosphate (dITP) in combination with DIG-deoxyuridine5 -triphosphate (DIG-dUTP) as the substrate for TdT tailing. The efficiency of labeling and the specificity of hybridization of the probe were evaluated in comparison with probes tailed with dATP in combination with DIG-dUT

\section{MATERIALS AND METHODS}

\section{Materials}

dITP was purchased from Sigma (St. Louis, MO, USA). Positively charged nylon membranes were purchased from Roche Molecular Biochemicals (Mannheim, Germany). A synthetic oligonucleotide (5'-CCCTACAAAGAAAATGGAGAGCCT-3') to detect the testis-enhanced gene transcript (TEGT) gene was designed using an HYBsimulator ${ }^{\mathrm{TM}}$ (Advanced Gene Computing Technologies, Irvine, CA, USA). For the colony hybridization experiment, 275 types of synthetic oligonucleotides were designed. Synthetic oligonucleotides were purchased from Life Technologies (Gaithersburg, MD, USA). Plasmid DNA containing cDNA clones was obtained from a cDNA library (11).

\section{Tailing of Oligonucleotide Using TdT}

Oligonucleotide probes were tailed with DIG-labeled nucleotide using TdT 